\title{
INOVA
}

Working Paper

\# 594

2015

\section{Price Distortion under Fixed-Mobile Substitution}

\section{Marc Bourreau \\ Carlo Cambini \\ Steffen Hoernig}

NOVA

School

of Business

\& Economics

powerful

minds

7 7rivition 


\title{
Price Distortion under Fixed-Mobile Substitution
}

\author{
Marc Bourreau*, Carlo Cambini†, Steffen Hoernig ${ }^{\ddagger}$
}

29 June 2015

\begin{abstract}
This paper analyses the impact of substitution between fixed and mobile telephony on call prices. We develop a model where consumers differ in the benefits of mobility and firms price discriminate between on-net and off-net calls. We find that call prices are distorted downwards due to substitution possibilities and customer heterogeneity, and that this distortion increases with the fixed-mobile termination mark-up.
\end{abstract}

Keywords: Network competition; fixed-mobile substitution; price discrimination.

JEL codes: L51, L92.

${ }^{*}$ Telecom ParisTech, Department of Economics and Social Sciences, and CREST-LEI, Paris. Email: marc.bourreau@telecom-paristech.fr.

${ }^{\dagger}$ Politecnico di Torino, DIGEP; EUI - Florence School of Regulation. Email: carlo.cambini@polito.it.

${ }^{\ddagger}$ Corresponding author. Nova School of Business and Economics, Universidade Nova de Lisboa; CEPR, UK. Email: shoernig@novasbe.pt. Postal address: Nova SBE, Campus de Campolide, 1099-032 Lisboa, Portugal; tel. +351-213801600, fax +351-213870933. 


\section{Introduction}

Over the last 25 years mobile telephony has dramatically changed consumers' usage of communication services, as well as altered the historical market structure. Wireless services have substantially grown in terms of subscribership, revenues, and usage at the expenses of fixed ones. ${ }^{1}$ However, recent evidence shows that the majority of users still prefers to multi-home, i.e. adopt both mobile and fixed connections. The association of European telecoms regulators (BEREC, 2011) reported that although the share of mobile-only households increased and that of fixed-only households decreased over time, having both is now the most common situation in Europe and the share of multihomers is not declining over time. The most recent data from the Eurobarometer (2014) "E-communications survey" prepared for the European Commission shows that $61 \%$ of households in the EU27 have both fixed and mobile telephone access, around $31 \%$ only have mobile phones and $6 \%$ only have a fixed phone. ${ }^{2}$ Interestingly, the rate of mobileonly households varies considerably across Member States, with values ranging from $85 \%$ in Finland, $83 \%$ in the Czech Republic, to $55 \%$ in Denmark and $43 \%$ in Italy, or to values below the EU average in countries like Spain (28\%), UK (17\%), France (13\%), Germany $(8 \%)$ or Sweden $(2 \%)$.

The success of the mobile sector is to a large part due to fixed-mobile substitution. Major technological advances and cost reductions have enabled mobile carriers to decrease the difference between fixed and mobile prices, allowing them to become strong competitors to traditional fixed providers. Empirical studies have tried to quantify fixed-mobile substitution and its economic impact (see the surveys by Vogelsang, 2010 and 2015), but there is a lack of theoretical investigation the effect of consumers' preference for mobility on fixed-mobile substitution and on retail call prices. This paper tries to fill this gap and aims to point out how fixed-mobile substitution, and the consumer preference towards mobility, can distort retail prices of mobile services. This analysis would help policy makers to understand the effect of fixed-mobile substitution on mobile operators' pricing strategies and to better define the boundaries between previously separated fixed and

\footnotetext{
${ }^{1}$ See, for more details, Gruber and Verboven (2001).

${ }^{2}$ The distribution of different types of access has changed slightly over the past two years. The proportion of households with mobile access only has increased by 4 percentage points since $2011(31 \%$ vs. $27 \%$ ) while that of households with fixed access only has decreased by 3 percentage points. ( $6 \%$ vs. $9 \%)$.
} 
mobile communication markets.

From the theoretical point of view, there exists a relevant - though not large - literature on the relationship between fixed and mobile telephony, with a focus on the role of fixed-to-mobile access (or termination) charges. For example,Wright (2002), Valletti and Houpis (2005), and Baake and Mitusch (2009) consider fixed-to-mobile calls and focus on the impact of mobile termination charges on retail charges and the number of users. These papers however do not consider the role of fixed-mobile substitution (both at access and service level), and more importantly, they do not take into account consumer heterogeneity with respect to the benefits for mobility. Moreover, they focus on the effect of access charges on market equilibria, while our main focus is on retail price distortion.

Armstrong and Wright (2009) also analyze the role of call substitution but their focus is on the role of voluntary vs. regulated setting of termination rates. These authors show that direct substitution between fixed- and mobile-originated calls weakens the competitive bottleneck of call termination. Hence, the welfare gains from regulating mobile termination rates are smaller, while private incentives do not imply excessively high levels. Our paper departs from this study by analyzing the more realistic situation where substitution affects not only the types of calls made but also consumers' subscription decisions, though we do not endogenize the role of the termination charge. Our model closely considers the suggestion by Armstrong and Wright (2009) who state that "an alternative kind of substitution between fixed and mobile networks may take place at the subscription level rather than the per-call level, so that some people might give up their fixed line altogether and become mobile-only users." (p. 296).

Our model considers both fixed-mobile substitution and elastic subscription demands on both types of (fixed and mobile) networks. In particular, drawing on our previous paper (Hoernig et al., 2015), we develop a game-theoretic model with three independent but interconnected operators, one fixed and two mobile networks. On the demand side, all subscribers spend the same fraction of their time outside home, but each of them has a different personal benefit from having a mobile phone. Depending on their benefit for mobile communications, subscribers choose whether to single-home or to multi-home, that is, whether to subscribe to a fixed or a mobile network, or to both. This implies that fixed and mobile telephony are simultaneously substitutes and complements: They are complements because even owners of a fixed line benefit from having a mobile sub- 
scription, by being able to make calls when they are not at home. They are substitutes because when both networks are available only the cheaper type of call will be used.

This paper differs from our companion paper in two ways. First, the goal of Hoernig et al. (2015) was to analyze the impact of varying levels of termination rates on consumers' subscription decisions. The key question was whether the different regulatory treatment of termination on fixed and mobile networks affects the development of fixed and mobile subscription decisions. Our previous findings show that termination rates have an effect on subscription substitution and fixed disconnection: A higher mobile (fixed) termination rate increases the number of mobile (fixed) users and lowers that of fixed (mobile) users, via the waterbed and cost effects, respectively. Higher termination rates also tend to increase the number of customers who do not adhere to any network, identifying a tradeoff between the uptake of individual networks and total uptake.

The present paper is centered instead on the setting of retail prices, and not on wholesale prices, and points out the kind of retail level distortions mobile operators could adopt to gain market share in the presence of fixed-mobile substitution. Second, and more importantly, in this paper we use a more realistic retail pricing structure close to the one adopted in reality by mobile operators. In particular, we assume that firms adopt multi-part tariffs with network-based price discrimination (i.e., call charges depend on the destination network). For calls only between mobile networks, this pricing scheme has been already adopted in several other papers, such as Laffont, Rey and Tirole (1998), Gans and King (2001), Cambini and Valletti (2003), Hoernig (2007 and 2014), but none of these studies takes into account the benefit from mobility and the presence of fixed-mobile substitution as we do in this paper.

The focus on fixed-mobile substitution is not only theoretically interesting in itself since it contributes to the previous literature, but it is also founded on the recent empirical literature. Indeed, recent studies on EU data provide stronger evidence on fixed-mobile call substitution across European countries. Briglauer et al. (2011) find that, at least for Austria, fixed and mobile calls are strong substitutes while access substitution is rather weak. Grzybowski (2014) analyzes substitution between access to fixed-line and mobile telephony in the European Union and shows that decreasing prices for mobile services increases the share of mobile-only households and decreases the share of fixedonly and fixed-mobile households, which suggests substitution between fixed-line and 
mobile connections. Finally, Grzybowski and Verboven (2014) show that fixed and mobile connections are generally perceived in the EU as substitutes, especially in regions with a higher GDP per capita, but also that there is a lot of heterogeneity across countries due to the interplay of specific social and economic features (i.e., households with different age, education, professional activity, etc.). However, all these papers do not empirically consider the presence of network based price discrimination, since they generally consider an average mobile charge without looking at single price components (i.e. on-net and off-net prices) separately. Hence, this paper also provides new testable predictions for future empirical work on the impact of fixed-mobile substitution on mobile commercial tariffs.

Our model predicts that mobile call prices for on-net, off-net and mobile-to-fixed calls are all distorted downwards due to substitution possibilities and this distortion is greater the higher is the fixed-mobile termination mark-up. This implies that mobile networks adapt their pricing structure to compete stronger for mobile-only customers than for fixed-mobile customers. Indeed, our results show that fixed-mobile substitution generates a waterbed effect between fixed-to-mobile and mobile-only profits: the higher profits from fixed-to-mobile termination are used to cut mobile-only retail prices and enhance competition between different technologies.

The paper is organized as follows. In Section 2 we first lay out the model, and then discuss the equilibrium tariffs in Section 3. Finally, we conclude.

\section{Model Setup}

Consumers and firms. We assume that there are two mobile networks and one independent fixed network. ${ }^{3}$ In order to concentrate on the distortions in mobile call prices, we assume that call prices on the fixed network are equal to the corresponding marginal cost, including termination rates, which tends to result from multi-part tariffs.

A mass 1 of consumers decides whether to subscribe to a mobile network and/or to the fixed network. According to network choice, we call them mobile-only (M), fixed-only $(\mathrm{F})$, and fixed-mobile (FM) subscribers. Consumers spend a fraction of their time $\lambda \in(0,1)$

\footnotetext{
${ }^{3}$ In a companion paper, Hoernig et al. (2015), we assume uniform pricing on mobile networks and focus on the effects of termination rates.
} 
outside home. When on the move, which happens with probability $\lambda$, a consumer can only use a mobile phone to make or receive calls. When at home the consumer may have access to a fixed and/or a mobile phone; if he has both, he chooses whichever is cheaper to make calls.

Calling patterns are balanced, and consumers are on the move or at home independently of each other. ${ }^{4}$ They are heterogenous in their fixed benefit from mobility and, independently, in their preferences for the two mobile networks, as we will specify below.

We further assume that when consumers choose which network(s) to join, they know their benefits from mobility, but not yet their network preference. For example, consumers may first decide whether or not to have a mobile phone and which one (e.g., an iPhone or an Android phone), and then only decide which mobile operator to subscribe to. ${ }^{5}$

Mobile operator $i$ 's numbers of M- and FM-clients are $\mu_{i}^{m}$ and $\mu_{i}^{m x}$, respectively, for $i=1,2$. Its total number of subscribers is then $\mu_{i}=\mu_{i}^{m}+\mu_{i}^{m x}$. The total numbers of M- and FM-clients are $\mu^{m}=\mu_{1}^{m}+\mu_{2}^{m}$ and $\mu^{m x}=\mu_{1}^{m x}+\mu_{2}^{m x}$, respectively, the total number of mobile subscribers is $\mu=\mu^{m}+\mu^{m x}$, and there are $\mu^{x}$ fixed clients. We assume that consumers and the mobile operators have rational expectations about the number of subscribers for each network technology $\left(\mu^{m}, \mu^{m x}\right.$ and $\left.\mu^{x}\right)$. That is, they take them as given when they make their subscription and tariff decisions, while in the end their value must be consistent with the resulting equilibrium tariffs and subscriber numbers. On the other hand, mobile operators take into account that their individual market shares $\mu_{i}^{m}$ and $\mu_{i}^{m x}$ are affected directly by tariff decisions, while consumers have rational expectations $\bar{\mu}_{i}^{m}$ and $\bar{\mu}_{i}^{m x}{ }^{6}$ We will of course assume that in equilibrium expectations coincide with realized values, i.e. $\bar{\mu}_{i}^{m}=\mu_{i}^{m}$ and $\bar{\mu}_{i}^{m x}=\mu_{i}^{m x}$.

Mobile networks incur a fixed cost $f$ per subscriber, marginal costs of origination and termination $c_{o}$ and $c_{t}$, and on-net $\operatorname{costs} c=c_{o}+c_{t}$. In order to determine the determinants of equilibrium prices, we allow for mobile termination rates that depend on the origin of calls: For mobile-to-mobile calls, it is $a=c_{t}+m$, and for fixed-to-mobile calls it is $a_{f m}=c_{t}+n$, where $m, n \geq 0$ are the corresponding termination margins. We will see

\footnotetext{
${ }^{4}$ We ignore intertemporal substitution, that is, the possibility for consumers that are on the road to postpone calls until they arrive at home.

${ }^{5}$ This timing assumption leads to simple expressions for market shares and keeps the analysis technically feasible.

${ }^{6}$ We also analysed the model setup where $\mu^{m}, \mu^{m x}$ and $\mu^{x}$ vary with out-of-equilibrium tariffs (rather than only equilibrium tariffs), and show that the results are qualitatively similar though much more complicated mathematically.
} 
below that retail pricing distortions exist if and only if $n>0$. Similarly, the fixed network has marginal costs $c_{x o}<c_{o}$ and $c_{x t}<c_{t}$, on-net cost $c_{x}=c_{x o}+c_{x t}$, and a termination rate $a_{x}=c_{x t}+m_{x}, m_{x} \geq 0$.

We make the assumption that the fixed-to-mobile termination margin is small, $n<$ $c_{o}-c_{x o} \cdot{ }^{7}$ As a result, the marginal costs of calls are ordered as follows:

$$
\begin{aligned}
c_{x o}+c_{x t} & <c_{o}+c_{x t}+m_{x}, \\
c_{x o}+c_{t}+n & <c_{o}+c_{t}<c_{o}+c_{t}+m .
\end{aligned}
$$

Tariffs and surplus. Mobile network $i$ charges a tariff $\left(F_{i}, p_{i}, \hat{p}_{i}, p_{i x}\right)$, where $F_{i}$ is a monthly fixed fee, and $p_{i}, \hat{p}_{i}$ and $p_{i x}$ are the mobile-to-mobile on-net, off-net and mobileto-fixed per-minute call prices, respectively. As mentioned above, the fixed network sets call prices equal to their marginal cost, on-net $p_{x}=c_{x o}+c_{x t}$ and fixed-to-mobile $p_{x m}=c_{o x}+a_{f m}$. We also postulate (and later confirm), that the following ordering of call prices holds:

$$
p_{x}<p_{i x}, p_{x m}<p_{i}<\hat{p}_{i}
$$

which implies that mobile calls are only used when no fixed phone is available. Note also that due to (1), this ordering of prices holds when equilibrium prices are close enough to their respective marginal cost.

The consumption utility from a call of length $q$ is $u(q)$, where $u^{\prime}>0$ and $u^{\prime \prime}<0$. For price $p$, the caller's indirect utility is $v(p)=\max _{q} u(q)-p q$, and the call duration is $q=-v^{\prime}(p)$, leading to the obvious short-hands $v_{i}=v\left(p_{i}\right), \hat{v}_{i}=v\left(\hat{p}_{i}\right)$, etc. Apart from the surplus obtained from making calls, subscribers obtain an access benefit that depends on the network(s) they subscribe to. If a consumer subscribes only to a mobile network or to the fixed network, his subscription benefit is $A_{m}$ or $A_{x}$, respectively. If he subscribes to both types of networks, he obtains an access benefit of $A_{m x}$.

A mobile-only user makes all calls with his mobile phone and obtains the surplus

$$
w_{i}^{m}=A_{m}-F_{i}+\bar{\rho}_{i}^{m} v_{i}+\bar{\rho}_{j}^{m} \hat{v}_{i}+\rho^{x} v_{i x}
$$

\footnotetext{
${ }^{7}$ It can be shown that for $n>c_{o}-c_{x o}$ the mobile on-net price is not distorted, while qualitatively similar distortions are found for the mobile off-net price and the mobile-to-fixed price.
} 
where $\rho_{l}^{m}=\mu_{l}^{m}+\lambda \mu_{l}^{m x}$ denotes the number of receivers on mobile network $l=i, j$, $\bar{\rho}_{l}^{m}=\bar{\mu}_{l}^{m}+\lambda \bar{\mu}_{l}^{m x}$ the corresponding consumer expectation, and $\rho^{x}=(1-\lambda)\left(\mu^{m x}+\mu^{x}\right)$ the number of receivers on fixed phones.

Similarly, a fixed-only user makes all calls from his fixed line, and receives the surplus

$$
w^{x}=A_{x}-F_{x}+(1-\lambda)\left(\rho^{m} v_{x m}+\rho^{x} v_{x}\right),
$$

where $\rho^{m}=\mu^{m}+\lambda \mu^{m x}$. For further reference below, also define $\rho^{\lambda}=\mu^{m}+\lambda^{2} \mu^{m x}$ and $\Phi=\left(\rho^{\lambda} / \rho^{m}\right)-\left(\rho^{m} / \mu\right)>0$. Note that fixed-only users can only make calls when they are at home, which occurs with probability $1-\lambda$.

When an FM-subscriber of network $i$ is on the move, the order of prices (2) implies that it is cheaper for him to make mobile-to-fixed calls than mobile-to-mobile calls when receivers are at home $\left(p_{i x}<p_{i}, \hat{p}_{i}\right)$. Thus, mobile-to-mobile calls are only made when receivers are themselves on the move. ${ }^{8}$ Similarly, when he is at home it is cheaper to use the fixed phone than the mobile phone for any type of calls. We can then express the expected surplus of FM-subscribers as

$$
w_{i}^{m x}=A_{m x}-F_{i}-F_{x}+\lambda\left[\bar{\rho}_{i}^{m} v_{i}+\bar{\rho}_{j}^{m} \hat{v}_{i}+\rho^{x} v_{i x}\right]+(1-\lambda)\left[\rho^{m} v_{x m}+\rho^{x} v_{x}\right]
$$

There is substitution to cheaper calls involving the fixed network both when callers are on the road and when they are at home.

Market shares. As already mentioned, consumers differ in their preferences for mobile networks. They learn these preferences after deciding to take up a mobile subscription, but before choosing a specific mobile network. For the purpose of this paper, we assume that the former decision has already been taken, ${ }^{9}$ thus we consider the subscriber numbers $\mu^{m}, \mu^{m x}$ and $\mu^{x}$ as fixed, while mobile operators still compete for their share of consumers. More precisely, we assume that mobile operator $i$ 's market shares of M- and

\footnotetext{
${ }^{8}$ We can imagine that the caller first calls the receiver's fixed line, and then calls him on his mobile if he is not at home.

${ }^{9}$ This assumption does not affect the analysis of equilibrium call prices. In our companion paper we endogenize this decision.
} 
FM-subscribers are given by

$$
\mu_{i}^{m}=y\left(w_{i}^{m}-w_{j}^{m}\right) \mu^{m} \text {, and } \mu_{i}^{m x}=y\left(w_{i}^{m x}-w_{j}^{m x}\right) \mu^{m x}
$$

respectively, where $y$ is a function $y: \mathbb{R} \rightarrow[0,1]$, with $y(0)=1 / 2, y^{\prime}>0$ and $y^{\prime}(0)=\sigma>$ 0 . An often-used special case is the Hotelling model, which corresponds to $y(x)=1 / 2+\sigma x$ (over the relevant range).

Profits. The profits of mobile network $i$ are given by

$$
\begin{aligned}
\pi_{i}= & \mu_{i}\left(F_{i}-f\right)+\rho_{i}^{m}\left[\rho_{i}^{m}\left(p_{i}-c\right) q_{i}+\rho_{j}^{m}\left(\hat{p}_{i}-c-m\right) \hat{q}_{i}\right. \\
& \left.+\rho^{x}\left(p_{i x}-c_{o}-a_{x}\right) q_{i x}+\rho_{j}^{m} m \hat{q}_{j}+\rho^{x} n q_{x m}\right] .
\end{aligned}
$$

They consist of profits from subscriptions, from calls to other mobile users, from calls to the fixed network, and termination profits from calls arriving from the other mobile and the fixed network.

\section{Equilibrium Tariffs}

We now determine the symmetric equilibrium tariff $\left(F_{i}, p_{i}, \hat{p}_{i}, p_{i x}\right)$ of mobile operators as a Nash equilibrium in their pricing game. The proof of the following Proposition is in the Appendix. ${ }^{10}$

Proposition 1 In the symmetric (rational expectations) Nash equilibrium of the mobile market, we have the following outcomes:

1. Mobile call prices are set below their respective marginal costs if and only if the fixed-to-mobile termination margin is positive (i.e. $n>0$ ),

$$
\begin{aligned}
p_{i}^{*} & =c+n\left(q_{i} / q_{i}^{\prime}\right) \Omega \\
\hat{p}_{i}^{*} & =c+m+n\left(\hat{q}_{i} / \hat{q}_{i}^{\prime}\right) \Omega, \\
p_{i x}^{*} & =c_{o}+a_{x}+n\left(q_{i x} / q_{i x}^{\prime}\right) \Omega,
\end{aligned}
$$

\footnotetext{
${ }^{10}$ We assume the existence of a shared equilibrium. This is ensured as long as $\sigma$ is low enough and a is close to the marginal transportation cost. The proof of existence would follow the same lines of Laffont et al. (1998), as extended to asymmetric settings by de Bijl and Peitz (2002).
} 
where

$$
\Omega=\frac{\rho^{x} q_{x m}}{1 /(2 \sigma \Phi)-\rho^{m} q_{i}^{2} / q_{i}^{\prime}-\rho^{x} q_{i x}^{2} / q_{i x}^{\prime}}>0 ; \Phi=\frac{\mu^{m}+\lambda^{2} \mu^{m f}}{\rho^{m}}-\frac{\rho^{m}}{\mu}>0
$$

2. fixed fees are equal to

$$
F_{i}^{*}=f+\frac{1}{2 \sigma}\left(1-n \frac{\rho^{m} \Omega}{\mu \Phi}\right)
$$

and equilibrium profits are

$$
\pi_{i}^{*}=\frac{\mu}{4 \sigma}+\frac{\left(\rho^{m}\right)^{2}}{4}\left\{n\left[\left(\hat{q}_{i}^{2} / \hat{q}_{i}^{\prime}\right)-\left(q_{i}^{2} / q_{i}^{\prime}\right)\right] \Omega+m \hat{q}_{i}\right\}
$$

Proposition 1 provides interesting and new results. First of all, since $q_{i}^{\prime}, \hat{q}_{i}^{\prime} q_{i x}^{\prime}<0$, all retail prices for on-net, off-net and mobile-to-fixed calls are distorted downwards. Interestingly, this distortion applies to all calls in the same direction. Indeed, in the previous literature on network competition with network based price discrimination (e.g. Laffont et al. 1998, Jeon et al. 2004), either all call prices are identical to marginal cost, or on-net call prices are set below marginal cost and off-net call price above.

Second, the retail price distortion is proportional to the mark up on fixed-to-mobile termination charges, $n$. Indeed, the above conditions show that the distortion disappears if and only if $n=0$, i.e. no profits can be made from terminating fixed-mobile calls.

The main intuition of this result is therefore as follows: FM-customers can be reached on both mobile and fixed phones, while M-customers can only be reached on their mobile phones. This implies that M-customers bring more valuable call termination business. This in turn leads mobile operators to decrease their own retail prices in order to compete more fiercely for mobile-only customers. Indeed, also the fixed fee $F_{i}^{*}$ decreases as $n$ gets larger. A waterbed effect is therefore in place: the profits from fixed-to-mobile termination are moved to decrease retail prices for calls which are proportionally more used by mobile-only customers.

It is interesting to note that the distortions in all call prices are qualitatively similar. Letting $\eta=p q^{\prime} / q$ denote the respective price elasticity of demand, we have:

$$
\frac{p_{i}-c}{p_{i}} \eta_{i}=\frac{\hat{p}_{i}-c-m}{\hat{p}_{i}} \hat{\eta}_{i}=\frac{p_{i x}-c_{o}-a_{x}}{p_{i x}} \eta_{i x}=n \Omega
$$


This underlines that the same strategic interaction determines the respective (negative) mark-ups.

The distortion due to fixed-mobile substitution depends also on $\Omega$. It is interesting to observe that this factor tends to decrease in $\lambda$, that is, the probability that consumers are on the move. This implies that the more mobile-only consumers are on the move, the lower is the distortion in call prices, everything else equal. Unfortunately, it is not possible to provide a more precise statement without introducing specific assumptions about the demand function. However, this result holds at least if mobile subscriptions are sufficiently heterogenous (i.e. low $\sigma$ ), which magnifies the effect of $\lambda$ on $\Phi$ and in turn on $\Omega$.

\section{Welfare Considerations}

Finally, it is important to consider the potential welfare effects of mobile price distortions emerging due to fixed-to-mobile substitution.

Given that subscriber numbers of different technologies are fixed, the equilibrium allocation depends only on call quantities. Call prices are below marginal costs including termination rates, but in the absence of call externalities (see Jeon et al., 2004) welfare would be maximized with call prices at network marginal cost. From Proposition 1 we observe that on-net prices are always too low, i.e. they are always below network marginal cost. This implies that too many on-net calls are made. In this setting, a high termination charge for the fixed-to-mobile calls benefits mobile users while reducing the consumer surplus of fixed telephony users (although some consumers use the two services). To restore efficiency, the socially optimal choice would be to reduce the margin for fixedto-mobile calls to zero, that is set fixed-to-mobile termination equal to marginal costs, i.e. $n=0$. With $n=0$ on-net price becomes socially optimal while the prices of off-net calls and mobile-to-fixed calls would remain above marginal costs as long as $m>0$ and $a_{x}$ is above cost. To further increase social welfare and fully internalize network externalities, then, the regulator might also set $m=0$ and $a_{x}$ at cost. The latter decision, though, is totally unrelated to the distortion due to fixed-mobile substitution we find in this paper, since the distortion is only driven by the fixed-to-mobile termination mark up $n$.

In a nutshell, in order to increase welfare and limit the price distortion that emerges 
due to fixed-mobile substitution, a possible regulatory intervention would be to move towards cost-oriented fixed-to-mobile termination. This reduction would mostly benefit fixed users that will pay less for any fixed-to-mobile call; on the contrary, mobile users will face a reduction of their surplus since they will no longer benefit from the "distorted" mobile tariffs. Further benefits may even come from eliminating any mark-ups from mobile-to-mobile and mobile-to-fixed termination charges. In this latter case, all consumers would benefit from a complete convergence between fixed and mobile calls and prices will be no longer distorted.

From a technological perspective, the distinction between fixed and mobile termination could also be much reduced in the context of forthcoming NGN/IP-based networks. In future integrated IP-based mobile and fixed networks, marginal costs for terminating a call will converge on both networks and approach zero. Moreover, voice will represent only a small fraction of traffic; therefore, total costs will have little to do with minutes of voice use, but will be much more driven by capacity requirements. The discrepancy between fixed and mobile termination rates appears therefore unjustified in a convergent market, even though the ratio between mobile (i.e. fixed-to-mobile $n$, and mobile-tomobile $m$ ) and fixed terminations rates has decreased recently. A report by BEREC (2013) shows that the average termination rate in the EU27 in January 2013 was equal to $2.58 €$ cents/minute, while the average fixed termination rate ranged between 0.50 and $0.80 €$ cents/minute according to the level (layer) of interconnection, still a ratio of 3.1. Hence, termination on mobile networks, and in particular fixed-to-mobile termination charges, is still at least three times more expensive than on fixed networks. Interestingly, this discrepancy is absent in the US where the ratio between fixed and mobile termination rates is 1:1. Indeed, in the US call termination has a strong tendency toward symmetry in the rates charged for reciprocal compensation, and the latter are capped at a rate that reflects the marginal cost of the fixed termination. In most of the negotiations mobile-to-mobile termination charges are set to zero (i.e. the so called "bill-and-keep"; Marcus, 2004; Vogelsang, 2015). This regulatory intervention might be useful not only to decrease retail prices overall, but also to avoid call price distortions due to fixed-mobile substitution as we highlight in this paper. 


\section{Conclusions}

In the last two decades mobile telephony has had a tremendous impact on the fixed telephony market. Due to the benefit from mobility, subscribers in many countries now use their mobiles more than their fixed lines, and quite often disconnect the latter. In this paper, we have presented a model that captures the substitution between fixed and mobile telephony at the subscription and call level, by taking into account consumer mobility (i.e., the fact that consumers are sometimes at home, and sometimes on the road). The main insight of the paper is that in a context of subscription substitution mobile networks distort downwards call prices for on-net, offnet and mobile-to-fixed calls. This distortion is greater the higher is the fixed-to-mobile termination mark-up. The intuition of this result is that mobile operators want to cut the retail prices of mobile-only users in order to attract a larger fraction of them, because this type of consumers generates more termination revenues than the consumers that use both mobile and fixed telephony. Thus, mobile networks adjust their retail prices and the fixed component of the tariff to compete stronger for mobile-only customers than for fixed-mobile customers. This is an instance of the waterbed effect, since the profits from fixed-to-mobile termination are moved to decrease retail prices for calls, which are proportionally more used by mobileonly customers.

This paper provides interesting findings to evaluate the regulatory design of mobile-tomobile and fixed-to-mobile termination rates over the last 20 years. Clearly, in the near future, the technological change towards all-IP networks will modify the scenario of the current analysis. It should lead to termination charges close to or at zero for both mobile and fixed networks. This model does not encompass such a setting but presents a kind of backward-looking analysis that is useful to highlight the impact that termination charges had, and still continue to have, on the phenomenon of fixed-to-mobile substitution. In the future, it seems likely that competition dynamics will be shaped by bundle offers with a reduced importance of paid-for voice calls (and thus weaker network effects at the operator level); substitution between fixed and mobile subscriptions will then mainly be driven by broadband quality. 


\section{References}

Armstrong, M. \& J. Wright (2009). "Mobile Call Termination." Economic Journal, 119(538), 270-307.

Baake, P. \& K. Mitusch (2009). "Mobile Phone Termination Charges with Asymmetric Regulation." Journal of Economics, 96, 241-261.

BEREC (2011), "BEREC report on impact of Fixed-Mobile substitution in market definition." BoR (11) 54, December.

BEREC (2013). "Termination Rates Benchmark Snapshot. Integrated Report on Mobile Termination Rates, Fixed Termination Rates \& SMS Termination Rates." BoR (13) 60, June.

Briglauer, W., Schwarz, A. \& C. Zulehner (2011). "Is fixed-mobile substitution strong enough to de-regulate fixed voice telephony? Evidence from the Austrian markets." Journal of Regulatory Economics, 39(1), 50-67.

Cambini C. \& T. Valletti (2003), "Network Competition with Price Discrimination: Billand-Keep is not so bad after all", Economics Letters, 81, 59-67.

De Bijl, P. and M. Peitz (2002), Regulation and Entry into Telecommunications Markets Cambridge University Press, Cambridge.

Eurobarometer (2014), "E-communications and telecoms single market household survey", Special Report 414, conducted by TNS Opinion \& Social at the request of Directorate-General for Communications Networks, Content \& Technology, Bruxelles.

Gans, J., \& King, S. (2001). "Using "bill and Keep" Interconnect Arrangements to Soften Network Competition." Economics Letters 71, 413-420.

Gruber H. \& F. Verboven (2001). "The Diffusion of Mobile Telecommunication Services in the European Union," European Economic Review, 45, 577-588.

Grzybowski, L. (2014). "Fixed-to-Mobile Substitution in the European Union." Telecommunications Policy, 38(7), 601-612. 
Grzybowski, L. \& F. Verboven (2014). "Substitution and Complementarity between Fixed-line and Mobile Access." Mimeo.

Jeon, D.S., Laffont, J.J. \& J. Tirole (2004). "On the Receiver-Pays Principle," RAND Journal of Economics, vol. 35(1), 85-110.

Hoernig S. (2007), "On-Net and Off-Net Pricing On Asymmetric Telecommunications Networks," Information Economics \&6 Policy, 19(2), 171-188.

Hoernig S. (2014), "Competition between multiple asymmetric networks: Theory and applications," International Journal of Industrial Organization, 32, 57-69.

Hoernig, S., Bourreau, M. \& C. Cambini (2015). "Fixed-Mobile Substitution and Termination Rates", Telecommunications Policy, forthcoming, http://dx.doi.org/10.1016/j.telpol.2014.12.005.

Laffont, J.-J., Rey, P. \& J. Tirole (1998), "Network Competition: II. Price Discrimination." RAND Journal of Economics, 29(1), 38-56.

Marcus, S.J. (2004), 'Call Termination Fees: the U.S. in Global Perspective', mimeo.

Valletti, T., \& G. Houpis (2005). "Mobile Termination: What is the 'Right' Charge?" Journal of Regulatory Economics, 28(3), 235-258.

Vogelsang, I. (2010). "The relationship between mobile and fixed-line communications: A survey." Information Economics and Policy, 22(1), 4-17.

Vogelsang, I. (2015), "Will the U.S. and EU Telecommunications Policies Converge? A Survey", Economia e Politica Industriale, 42(2), 117-155.

Wright, J. (2002). "Access pricing under competition: an application to cellular networks." Journal of Industrial Economics, 50, 289-315.

\section{Appendix}

Proof of Proposition 1: Take operator $j$ 's tariff as given. Mobile operator $i$ 's profits are $\pi_{i}=\mu_{i} T_{1}+\rho_{i}^{m} T_{2}$ with $T_{1}=F_{i}-f$ and

$$
T_{2}=\rho_{i}^{m}\left(p_{i}-c\right) q_{i}+\rho_{j}^{m}\left(\hat{p}_{i}-c-m\right) \hat{q}_{i}+\rho^{x}\left(p_{i x}-c_{o}-a_{x}\right) q_{i x}+\rho_{j}^{m} m \hat{q}_{j}+\rho^{x} n q_{x m} .
$$


The following derivatives are needed below, all originating from $\mu_{i}^{k}=y\left(w_{i}^{k}-w_{j}^{k}\right) \mu^{k}$, for $j \neq i$ and $k \in\{m, m x\}$ and given consumer expectations $\bar{\rho}_{i}^{m}$ and $\bar{\rho}_{j}^{m}$ :

$$
\begin{aligned}
\frac{\partial \mu_{i}^{m}}{\partial F_{i}} & =-\sigma \mu^{m}, \frac{\partial \mu_{i}^{m}}{\partial p_{i}}=-\sigma \mu^{m} \bar{\rho}_{i}^{m} q_{i}, \frac{\partial \mu_{i}^{m}}{\partial \hat{p}_{i}}=-\sigma \mu^{m} \bar{\rho}_{j}^{m} \hat{q}_{i}, \frac{\partial \mu_{i}^{m}}{\partial p_{i x}}=-\sigma \mu^{m} \rho^{x} q_{i x}, \\
\frac{\partial \mu_{i}^{m x}}{\partial F_{i}} & =-\sigma \mu^{m x}, \frac{\partial \mu_{i}^{m x}}{\partial p_{i}}=-\sigma \lambda \mu^{m x} \bar{\rho}_{i}^{m} q_{i}, \frac{\partial \mu_{i}^{m x}}{\partial \hat{p}_{i}}=-\sigma \lambda \mu^{m x} \bar{\rho}_{j}^{m} \hat{q}_{i}, \frac{\partial \mu_{i}^{m x}}{\partial p_{i x}}=-\sigma \lambda \mu^{m x} \rho^{x} q_{i x} .
\end{aligned}
$$

Thus, with $\rho^{m}=\mu^{m}+\lambda \mu^{m x}, \rho^{\lambda}=\mu^{m}+\lambda^{2} \mu^{m x}$, we have:

$$
\begin{aligned}
\frac{\partial \mu_{i}}{\partial F_{i}} & =-\sigma \mu, \frac{\partial \mu_{i}}{\partial p_{i}}=-\sigma \rho^{m} \bar{\rho}_{i}^{m} q_{i}, \frac{\partial \mu_{i}}{\partial \hat{p}_{i}}=-\sigma \rho^{m} \bar{\rho}_{j}^{m} \hat{q}_{i}, \frac{\partial \mu_{i}}{\partial p_{i x}}=-\sigma \rho^{m} \rho^{x} q_{i x} \\
\frac{\partial \rho_{i}^{m}}{\partial F_{i}} & =-\sigma \rho^{m}, \frac{\partial \rho_{i}^{m}}{\partial p_{i}}=-\sigma \rho^{\lambda} \bar{\rho}_{i}^{m} q_{i}, \frac{\partial \rho_{i}^{m}}{\partial \hat{p}_{i}}=-\sigma \rho^{\lambda} \bar{\rho}_{j}^{m} \hat{q}_{i}, \frac{\partial \rho_{i}^{m}}{\partial p_{i x}}=-\sigma \rho^{\lambda} \rho^{x} q_{i x}
\end{aligned}
$$

The first-order condition for profit maximization with respect to the fixed fee is

$$
0=\frac{\partial \pi_{i}}{\partial F_{i}}=\frac{\partial \mu_{i}}{\partial F_{i}} T_{1}+\mu_{i} 1+\frac{\partial \rho_{i}^{m}}{\partial F_{i}} T_{2}+\rho_{i}^{m}\left\{\frac{\partial \rho_{i}^{m}}{\partial F_{i}}\left(p_{i}-c\right) q_{i}+\frac{\partial \rho_{j}^{m}}{\partial F_{i}}\left(\hat{p}_{i}-c-m\right) \hat{q}_{i}+\frac{\partial \rho_{j}^{m}}{\partial F_{i}} m \hat{q}_{j}\right\}
$$

Assuming a symmetric equilibrium, this condition becomes

$$
0=T_{1}-\frac{1}{2 \sigma}+\frac{\rho^{m}}{\mu} T_{2}+\frac{\rho^{m} \rho^{m}}{2 \mu}\left\{\left(p_{i}-c\right) q_{i}-\left(\hat{p}_{i}-c-m\right) \hat{q}_{i}-m \hat{q}_{i}\right\}
$$

from which we obtain a preliminary form of equilibrium profits,

$$
\pi_{i}^{*}=\frac{\mu T_{1}+\rho^{m} T_{2}}{2}=\frac{\mu}{4 \sigma}-\frac{\left(\rho^{m}\right)^{2}}{4}\left\{\left(p_{i}-c\right) q_{i}-\left(\hat{p}_{i}-c-m\right) \hat{q}_{i}-m \hat{q}_{i}\right\}
$$

For the on-net price $p_{i}$, we have the first-order condition

$$
\begin{aligned}
0= & \frac{\partial \pi_{i}}{\partial p_{i}}=\frac{\partial \mu_{i}}{\partial p_{i}} T_{1}+\frac{\partial \rho_{i}^{m}}{\partial p_{i}} T_{2}+\rho_{i}^{m}\left\{\frac{\partial \rho_{i}^{m}}{\partial p_{i}}\left(p_{i}-c\right) q_{i}+\rho_{i}^{m} q_{i}+\rho_{i}^{m}\left(p_{i}-c\right) q_{i}^{\prime}\right. \\
& \left.+\frac{\partial \rho_{j}^{m}}{\partial p_{i}}\left(\hat{p}_{i}-c-m\right) \hat{q}_{i}+\frac{\partial \rho_{j}^{m}}{\partial p_{i}} m \hat{q}_{j}\right\},
\end{aligned}
$$

or, with symmetry and fulfilled expectations,

$$
0=T_{1}-\frac{1}{2 \sigma}+\frac{\rho^{\lambda}}{\rho^{m}} T_{2}+\frac{\rho^{\lambda}}{2}\left\{\left(p_{i}-c\right) q_{i}-\frac{p_{i}-c}{\sigma \rho^{\lambda} q_{i} / q_{i}^{\prime}}-\left(\hat{p}_{i}-c-m\right) \hat{q}_{i}-m \hat{q}_{j}\right\}
$$


For the off-net price $\hat{p}_{i}$, we have the first-order condition

$$
\begin{aligned}
0= & \frac{\partial \pi_{i}}{\partial \hat{p}_{i}}=\frac{\partial \mu_{i}}{\partial \hat{p}_{i}} T_{1}+\frac{\partial \rho_{i}^{m}}{\partial \hat{p}_{i}} T_{2}+\rho_{i}^{m}\left\{\frac{\partial \rho_{i}^{m}}{\partial \hat{p}_{i}}\left(p_{i}-c\right) q_{i}+\frac{\partial \rho_{j}^{m}}{\partial \hat{p}_{i}}\left(\hat{p}_{i}-c-m\right) \hat{q}_{i}\right. \\
& \left.+\rho_{j}^{m} \hat{q}_{i}+\rho_{j}^{m}\left(\hat{p}_{i}-c-m\right) \hat{q}_{i}^{\prime}+\frac{\partial \rho_{j}^{m}}{\partial \hat{p}_{i}} m \hat{q}_{j}\right\}
\end{aligned}
$$

or, with symmetry and fulfilled expectations,

$$
0=T_{1}-\frac{1}{2 \sigma}+\frac{\rho^{\lambda}}{\rho^{m}} T_{2}+\frac{\rho^{\lambda}}{2}\left\{\left(p_{i}-c\right) q_{i}-\left(\hat{p}_{i}-c-m\right) \hat{q}_{i}-\frac{\hat{p}_{i}-c-m}{\sigma \rho^{\lambda} \hat{q}_{i} / \hat{q}_{i}^{\prime}}-m \hat{q}_{j}\right\}
$$

For the mobile-to-fixed price $p_{i x}$ we have the first-order condition

$$
\begin{aligned}
0= & \frac{\partial \pi_{i}}{\partial p_{i x}}=\frac{\partial \mu_{i}}{\partial p_{i x}} T_{1}+\frac{\partial \rho_{i}^{m}}{\partial p_{i x}} T_{2}+\rho_{i}^{m}\left\{\frac{\partial \rho_{i}^{m}}{\partial p_{i x}}\left(p_{i}-c\right) q_{i}+\frac{\partial \rho_{j}^{m}}{\partial p_{i x}}\left(\hat{p}_{i}-c-m\right) \hat{q}_{i}+\rho^{x} q_{i x}\right. \\
& \left.+\rho^{x}\left(p_{i x}-c_{o}-a_{x}\right) q_{i x}^{\prime}+\frac{\partial \rho_{j}^{m}}{\partial p_{i x}} m \hat{q}_{j}\right\},
\end{aligned}
$$

or, with symmetry and fulfilled expectations,

$$
0=T_{1}-\frac{1}{2 \sigma}+\frac{\rho^{\lambda}}{\rho^{m}} T_{2}+\frac{\rho^{\lambda}}{2}\left\{\left(p_{i}-c\right) q_{i}-\left(\hat{p}_{i}-c-m\right) \hat{q}_{i}-\frac{p_{i x}-c_{o}-a_{x}}{\sigma \rho^{\lambda} q_{i x} / q_{i x}^{\prime}}-m \hat{q}_{j}\right\} .
$$

Combining (7), (8) and (9) with the first-order condition for the fixed fee (6) and the above expression for $T_{2}$ leads to the system

$$
\begin{aligned}
& 0=\rho^{m}\left(p_{i}-c\right) q_{i}+\rho^{x}\left(p_{i x}-c_{o}-a_{x}\right) q_{i x}+\rho^{x} n q_{x m}-\frac{p_{i}-c}{2 \sigma \Phi q_{i} / q_{i}^{\prime}} \\
& 0=\rho^{m}\left(p_{i}-c\right) q_{i}+\rho^{x}\left(p_{i x}-c_{o}-a_{x}\right) q_{i x}+\rho^{x} n q_{x m}-\frac{\hat{p}_{i}-c-m}{2 \sigma \Phi \hat{q}_{i} / \hat{q}_{i}^{\prime}} \\
& 0=\rho^{m}\left(p_{i}-c\right) q_{i}+\rho^{x}\left(p_{i x}-c_{o}-a_{x}\right) q_{i x}+\rho^{x} n q_{x m}-\frac{p_{i x}-c_{o}-a_{x}}{2 \sigma \Phi q_{i x} / q_{i x}^{\prime}}
\end{aligned}
$$

which is recursive in the second equation. Solving the first and third one first leads to equilibrium call prices

$$
p_{i}^{*}=c+n\left(q_{i} / q_{i}^{\prime}\right) \Omega, \hat{p}_{i}^{*}=c+m+n\left(\hat{q}_{i} / \hat{q}_{i}^{\prime}\right) \Omega, p_{i x}^{*}=c_{o}+a_{x}+n\left(q_{i x} / q_{i x}^{\prime}\right) \Omega,
$$

with

$$
\Omega=\frac{\rho^{x} q_{x m}}{1 /(2 \sigma \Phi)-\rho^{m} q_{i}^{2} / q_{i}^{\prime}-\rho^{x} q_{i x}^{2} / q_{i x}^{\prime}} .
$$


From (6) the fixed fee becomes

$$
\begin{aligned}
F_{i}^{*} & =f+\frac{1}{2 \sigma}-\frac{\rho^{m}}{\mu}\left\{T_{2}+\frac{\rho^{m}}{2}\left\{\left(p_{i}-c\right) q_{i}-\left(\hat{p}_{i}-c-m\right) \hat{q}_{i}-m \hat{q}_{i}\right\}\right\} \\
& =f+\frac{1}{2 \sigma}\left(1-n \frac{\rho^{m} \Omega}{\mu \Phi}\right)
\end{aligned}
$$

and profits are

$$
\pi_{i}^{*}=\frac{\mu}{4 \sigma}+\frac{\left(\rho^{m}\right)^{2}}{4}\left\{n\left[\left(\hat{q}_{i}^{2} / \hat{q}_{i}^{\prime}\right)-\left(q_{i}^{2} / q_{i}^{\prime}\right)\right] \Omega+m \hat{q}_{i}\right\}
$$




\section{INOVA}

\section{NOVA}

School

of Business

\& Economics

Shaping

powerful

minds

\section{Economics}

Nova School of Business and Economics

Faculdade de Economia

Universidade Nova de Lisboa

Campus de Campolide

1099-032 Lisboa PORTUGAL

Tel.: +351213801600

www.novasbe.pt 